\title{
DOS MODELOS SEMEJANTES DE NORIA DE TIRO
}

\author{
Luis RAMón-LACA MENÉndez de LuarCa
}

Real Jardín Botánico, C.S.I.C.

La noria de tiro es una máquina frecuente en el paisaje de las regiones secas de la Península Ibérica. Sólo en La Mancha se calcula que en los años veinte funcionaban cerca de veinte mil ${ }^{1}$. Hasta la mecanización de los sistemas de regadío fue el elemento clave para la extracción de agua de pozo con un caudal constante.

La mayor parte de los autores está de acuerdo en que el origen último de la noria de tiro se sitúa en Persia, aunque los árabes habrían actuado como difusores de un extremo a otro del Mediterráneo ${ }^{2}$. Desde Siria a la Península Ibérica las norias de tiro siguen un mismo modelo, por lo que no es de extrañar la sorpresa del embajador de Felipe II en la corte de Persia, donde vio unas ruedas como las que en Madrid usan [para regar] en los jardines ${ }^{3}$.

La procedencia árabe del vocabulario utilizado en la Península confirma la autoría de esta difusión. Todas las formas romances utilizadas para referirse a noria de tiro proceden del árabe. Además de las formas castellanas noria, de $n \bar{a}$ üra, y azud o azuda, de sudd, en árabe presa, en catalán aparece senia, de sāniya, en árabe noria de tiro ${ }^{4}$. Además, el árabe andalusí utilizaba dawlāb, única forma que no ha tenido descendientes 5 .

1 Glick, Th. F., «Noria pots in Spain». Technology and culture, 18, núm. 4, octubre 1977, pp. 644-650, p. 647. Cita a Th. Schioler, Roman and Islamic Water-lifting Wheels, Copenhagen, 1973, p. 16

2 Caro Baroja, J., Sobre la historia de la noria de tiro. En Tecnología popular española, pp. 351-407, Madrid, 1983, pp. 354-357.

3 Caro Baroja, J., op. cit., p. 372.

${ }^{4}$ En castellano sāniya dio aceña, según el DRAE molino harinero de agua situado dentro del cauce de un río.

5 También proceden del árabe los nombres de algunas piezas de la noria, como arcaduz, de $q \bar{a} d \bar{u}$ s. Sobre cuestiones filológicas veáse Colin, G. S., "La noria marocaine et les machines hydruliques dans le monde arabe», Hespéris, XIV (1932), pp. 23-60. 
A pesar de que la forma más usual en castellano es noria, en Toledo predominó la forma azuda (o localismos como zuda, zuba o zúa), lo que nos hace pensar que la utilización de un término u otro está más relacionada con la localidad que con razones tipológicas:

"(Açuda) es una rueda por estremo grande con que se saca agua de los rios caudalosos para regar las huertas. Destas maquinas ay muchas en la ribera del Tajo, cerca de Toledo" 6 .

Desde su introducción en la Península en la Edad Media, la noria de tiro se utilizó sin apenas diferencias hasta los años cuarenta o cincuenta de nuestro siglo. La única variación importante fue la sustitución de la madera por piezas de fundición a partir del siglo xIx. Hoy en día se conservan en buen estado una mínima parte, casi todas metálicas, y sólo muy recientemente comienzan a valorarse como un elemento importante del rico patrimonio etnográfico que los musulmanes dejaron en nuestro país en la cuestión del regadío.

\section{Azudas en la vega de Azucaica (Toledo)}

A juzgar por la cantidad de restos que se encuentran en la vega del Tajo, la noria debía de ser también muy abundante en La Sagra y los alrededores de Toledo. Los ejemplos que estudiamos en este trabajo, inéditos, nos van a ayudar a reconstruir el paisaje de una zona que, después de épocas de esplendor, hoy ha decaído completamente.

Se conservan en la vega de Azucaica, un pequeño pueblo situado a cinco kilómetros de Toledo, una porción de norias, albercas y acequias hoy desvinculadas de cualquier actividad agrícola. Sin embargo, una inspección cuidadosa de fotografías aéreas de esta zona revela una serie de estructuras enterradas dibujadas con bastante nitidez (fig. 1). Éstas podrían corresponder a antiguos cultivos, y si llevamos las cosas un poco al extremo quizá se trate de alguno de los huertos y casas de campo de los que habla el geógrafo medieval al-Idrīsī:

6 Covarrubias, S. de, Tesoro de la Lengua Castellana o Española, Madrid, 1611, p. 40. Aunque se refiere a norias de corriente, se aplica igualmente a noria de tiro. 


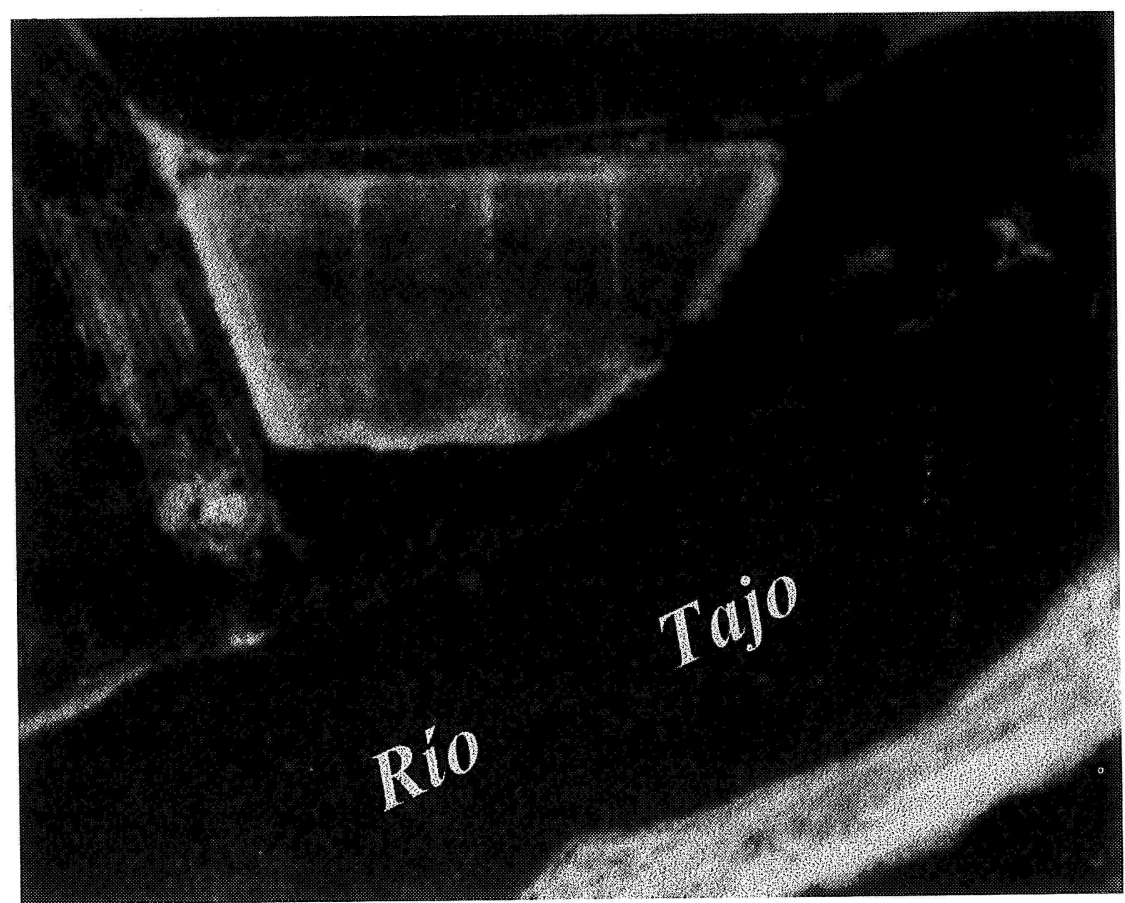

Figura 1: Un detalle de la vega de Azucaica a través de fotografía aérea.

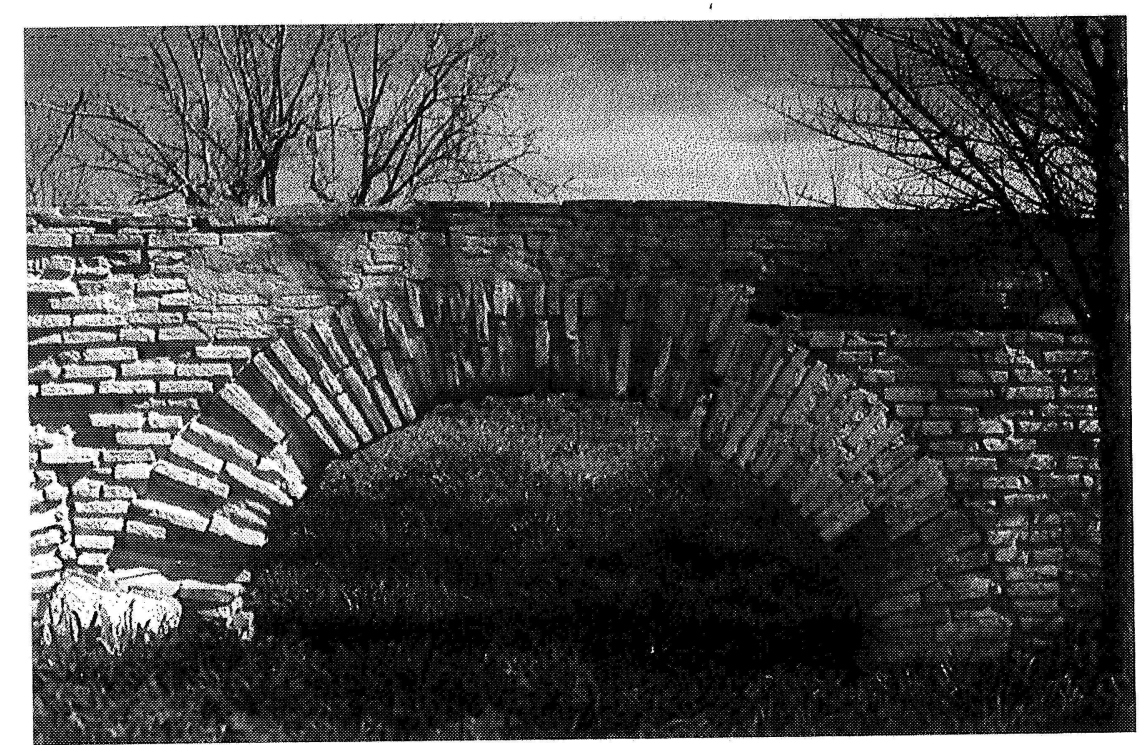

Figura 8: Arco del acueducto de La Noria. 


\begin{tabular}{|c|c|c|c|c|c|c|c|c|}
\hline $\begin{array}{l}\text { NOMBRES DE LAS } \\
\text { DEHESAS Y TERRENOS } \\
\text { IZQUIERDA DEL TAJO }\end{array}$ & 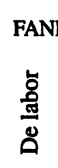 & 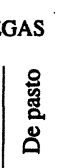 & $\begin{array}{c}\text { Olivas } \\
\text { que tienen }\end{array}$ & 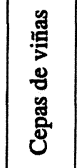 & 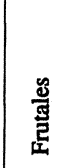 & Monte & Casas & Procedencias \\
\hline $\begin{array}{l}\text { Huertas del Rey } \\
\text { Cigarral del Carmen } \\
\text { Posesión de la Teja } \\
\text { Quinto de Horzagal } \\
\text { Alberquilla } \\
\text { Quinto de Aloquedo } \\
\text { Ramabujas } \\
\text { Ahin } \\
\text { DERECHA DEL TAJO }\end{array}$ & $\begin{array}{c}200 \\
" \\
" \\
96 \\
" \\
90 \\
540 \\
240\end{array}$ & $\begin{array}{r}" \\
" \\
78 \\
170 \\
526 \\
222 \\
1500 \\
660\end{array}$ & $\begin{array}{c}\Rightarrow \\
3000 \\
3000 \\
" \\
1010 \\
\Rightarrow \\
2584 \\
\Rightarrow\end{array}$ & $\mid \begin{array}{c}" \\
24000 \\
" \\
" \\
" \\
"\end{array}$ & $\begin{array}{l}" \\
" \\
" \\
" \\
" \\
" \\
" \\
"\end{array}$ & $\begin{array}{c}" \\
" \\
" \\
" \\
\text { Alameda } \\
\text { id. } \\
\text { id. } \\
\text { id. }\end{array}$ & $\begin{array}{c}1 \text { casa y } 1 \text { cas } \\
\text { Una casa } \\
1 \\
» \\
1 \\
» \\
1 \\
\text { Casa y cap." }\end{array}$ & $\begin{array}{l}\text { Vinculaciones } \\
\text { De concurso } \\
\text { Propiedad partic } \\
\text { Condado } \\
\text { Propiedad partic } \\
\text { Marquesado } \\
\text { Propiedad partic } \\
\text { Ducado }\end{array}$ \\
\hline $\begin{array}{l}\text { Pinedo y el Vinculo } \\
\text { Valdecubas } \\
\text { Villagomez } \\
\text { Mazarracin } \\
\text { La Peña } \\
\text { Casa de Campo } \\
\text { Azucaica } 1^{\mathrm{a}} \\
\text { Azucaica } 2^{\mathrm{a}}\end{array}$ & $\begin{array}{r}300 \\
370 \\
" \\
320 \\
75 \\
134 \\
96 \\
85\end{array}$ & $\begin{array}{c}900 \\
687 \\
" \\
880 \\
" \\
" \\
" \\
"\end{array}$ & $\begin{array}{c}1600 \\
132 \\
1500 \\
" \\
" \\
100 \\
" \\
1600\end{array}$ & $\begin{array}{l}\text { " } \\
" \\
" \\
" \\
" \\
" \\
"\end{array}$ & $\begin{array}{c}" \\
" \\
1000 \\
\text { Almen } \\
" \\
200 \\
" \\
700\end{array}$ & $\begin{array}{c}\text { Alto } \\
» \\
» \\
» \\
» \\
\text { Alameda } \\
» \\
\text { Alameda }\end{array}$ & $\begin{array}{l}1 \\
1 \\
1 \\
1 \\
" \\
1 \\
1 \\
1\end{array}$ & \begin{tabular}{l} 
Propiedad partic \\
Ducado \\
Propiedad partic \\
\multicolumn{1}{c}{ id. } \\
id. \\
Condado \\
Propiedad partic \\
Marquesado
\end{tabular} \\
\hline
\end{tabular}

Figura 2: Algunas dehesas de la vega del Tajo según P. Madoz, Diccionario GeográficoEstadístico-Histórico, t. XIV, Toledo, pp. 832-833, Madrid, 1849.

«Los jardines que rodean a Toledo están regados por canales, hay norias ${ }^{7}$ para el riego de las huertas, que producen en cantidad prodigiosa frutos de una belleza y una bondad extraña. Se admiran desde todos lados las bellas posesiones y los castillos fortificados» ${ }^{8}$.

Estas casas de campo son el núcleo de una serie de fincas y dehesas que se suceden a lo largo del Tajo (fig. 2). En este trabajo vamos a detenernos en dos de estas dehesas, Azucaica y La Alberquilla.

Sabemos que en 1095 Alfonso VI donaba la casa de campo de Zuqueyca al monasterio benedictino de San Servando ${ }^{9}$. Durante los siglos XII y XIII hay testimonio de la actividad de los mozárabes en la vega ${ }^{10}, \mathrm{y}$ dehesas como La Huerta del Rey, Ahín ('Ayn) y Mazarabuzaque (Manzil $A b \bar{u} I s h \bar{a} q)$ en la margen izquierda y Valdecubas, Azucaica ( $A l-S u$ -

7 Utiliza la forma dawälīb, plural de dawläb.

8 Al-Himyarī, La Péninsule ibérique au Moyen Âge d'après le Kitāb ar-Raw ạ al-Mi'tār, edic. y trad. al francés de E. Lévi-Provençal, Leiden, 1938, pp. 132-133.

9 Martín Gamero, A., Los cigarrales de Toledo, Toledo, 1857, p. 55.

10 Véase González Palencia, A., Los mozárabes de Toledo en los siglos XII y XIII, 4 vols., Madrid, 1926-1930. 
kayka) (fig. 3) y Mazarracín (Manzil Razin) en la derecha aparecen citadas en repetidas ocasiones. La noria aparece citada en Azucaica ya en 1149 , en un documento en el que se mencionan los pies de una noria antigua, aunque seguramente se trata a una noria de corriente ${ }^{11}$.

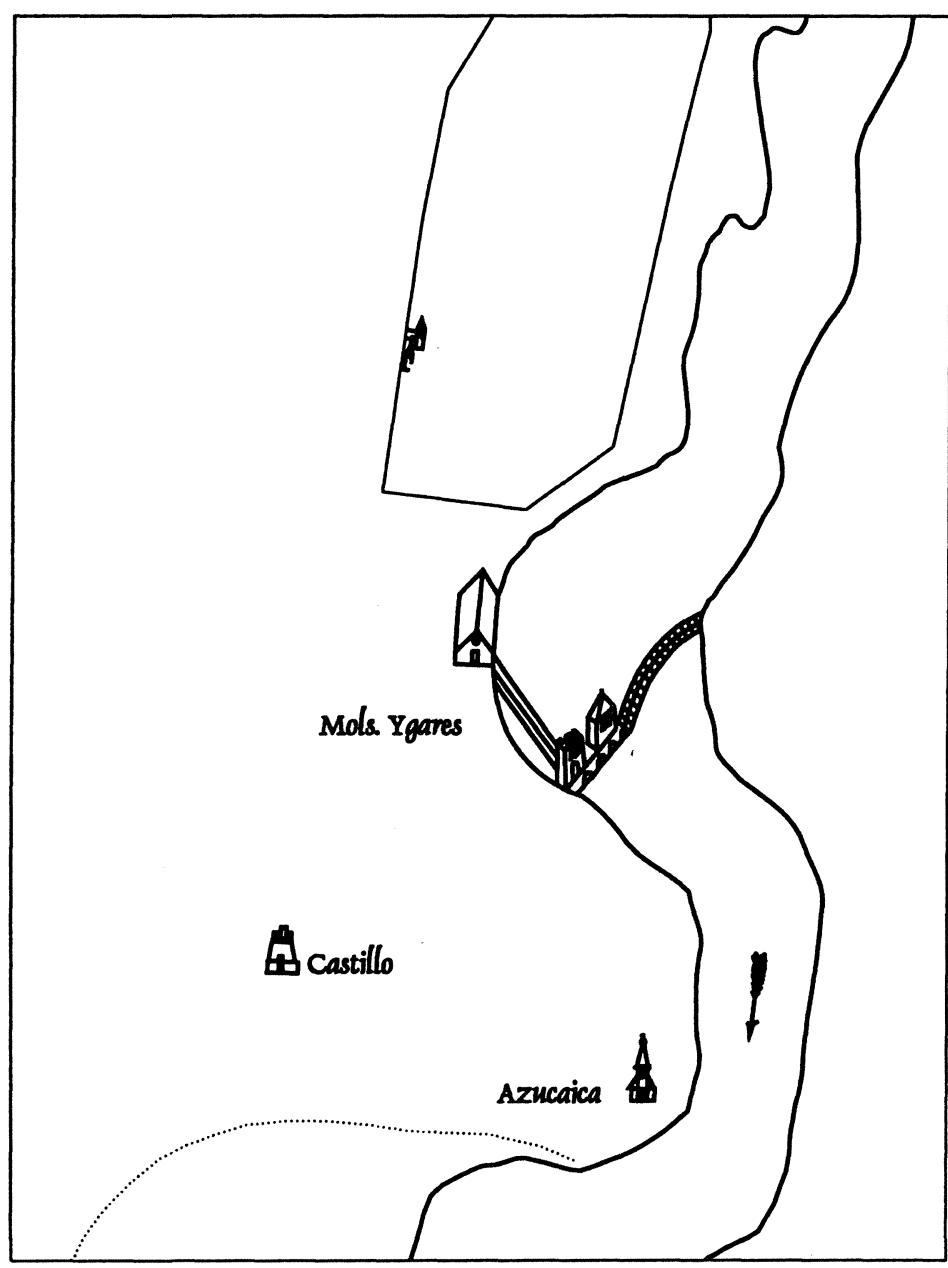

Figura 3: Azucaica en el siglo xIx. En Planos que representan los reconocimientos de las riberas del Tajo, p. 52, París, 1829.

11 Al-nā'üra al-qadìma, una noria antigua, González Palencia, A., op. cit., I, p. 109. 
En el siglo XVI los moriscos granadinos trabajaban en las haciendas de los alrededores de Azucaica, Velilla y Mocejón, y en este momento la zona debió recobrar buena parte de actividad. Los moriscos tenían fama de ser buenos horticultores y entendidos en la reparación de las norias:

"Vos el dicho Luis de Belmonte entraredes a trabajar en la dicha huerta ambos a dos a nuestra costa habemos de mondar y limpiar el paso de la noria que está en élla y si fuera necesario, sacarle los cantos que estén dentro del dicho pozo pegadas a las paredes y ahondarle alguna cosa para que tenga más abundancia de agua y si la dicha noria en todo el dicho tiempo tuviese necesidad de algún aderezo, sogas e arcaduces e otra cosan 12 .

Quizá se construyeran en esta época los ejemplos que vamos a analizar en este trabajo. En 1576 se habla de la reconstrucción de las casas de campo de Azucaica, por lo que es muy posible que las norias se construyeron al mismo tiempo:

«Una pequeña y hermosa población, llamada Açuqueyca, nombre arabe ques la junta de las caserias de los herederos del dicho soto, [próximo a Higares] de nuevo en este lugar se an labrado algunas buenas casas de morada y recreo, como son las del jurado Alonso Sanchez Hurtado, las del doctor Toro y otras que se van labrando» ${ }^{13}$.

En esta época había en la Huerta del Rey dos azudas llamadas de Razazul de Arriva y Razazul de Avajo distantes entre sí como beinte o beinte y quatro baras ${ }^{14}$.

En el siglo XVIII una hacienda llamada el Jaspe no tenía bien arreglados el instrumento de agua y pozos con que se regaba ${ }^{15}$, y por fin, en los planos catastrales elaborados entre los años 1936 y 1944 pueden contarse en esta zona de la vega unas cien norias, sólo en la parte de Azucaica unas treinta (fig. 4).

12 Magán García, J. M., y Sánchez González, R., Moriscos granadinos en La Sagra de Toledo 1570-1610, Toledo, 1993, p. 30.

13 Hurtado de Toledo, L., Memorial III. Relaciones de los pueblos de España ordenadas por Felipe II, edic. de C. Viñas y R. Paz. Toledo, 1951.

14 Archivo Municipal de Toledo, Plantios, Caja 2.

15 Larruga, E., Memorias politicas y económicas, V, pp. 205-206; Madrid, 1789. Citado por J. Caro Baroja en Norias, azudas, aceñas. En Tecnología popular española, pp. $239-348$, p. 326 . 


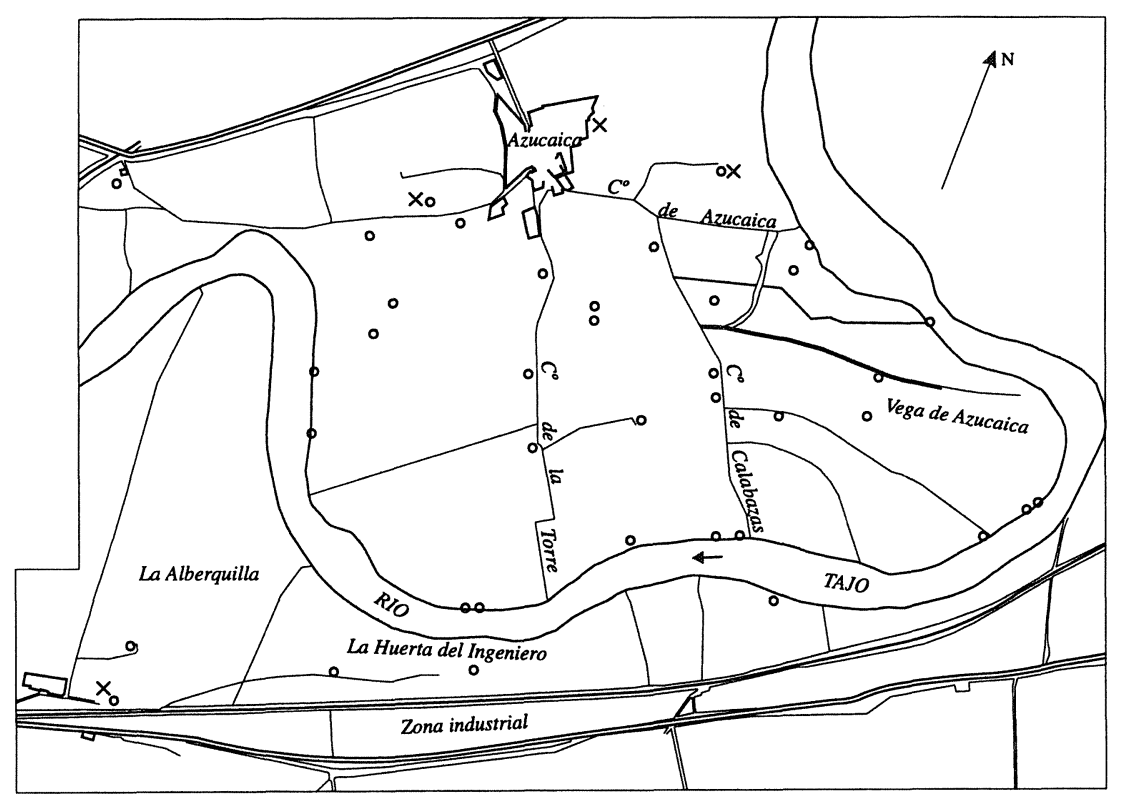

Figura 4: Norias en la Vega del Tajo, según Planos catastrales de 1936-1944 (originales e $1 / 5000)$. Cada o representa una noria $\mathrm{y} \times$ los ejemplos estudiados.

En Azucaica se recuerda una zuda en cada una de las decenas de casetas de pozos que se levantan en la vega. En la mayor parte de los casos sólo queda el hoyo donde estaba la rueda y los árboles que daban sombra a la maquinaria y al animal de tiro. Tradicionalmente, los árboles frutales y las moreras se plantaban en la vega con arreglo y a marco, en hilera, mientras que los olmos y álamos crecían alrededor de los andenes y alvercas de los pozos y norias sin estar con orden ${ }^{16}$. Esta cuestión nos ayuda a identificar los lugares donde existía una noria. Además de olmos, en algunos casos se plantaban también nogales, higueras y árboles del paraíso ${ }^{17}$.

La ciudad de Toledo ejercía un estricto control sobre este arbolado, puesto que para la tala de cada pie era necesario solicitar el permiso correspondiente de la Junta de Árboles, que solía concederlo si la

16 Toledo 1751 según las Respuestas Generales del Catastro del Marqués de la Ensenada, Madrid, 1990, pp. 40-41.

17 Cuando hablan de álamo negro, tanto los documentos antiguos como los vecinos actuales de Azucaica se están refiriendo al Ulmus minor L., no al Populus nigra L. 
petición estaba debidamente justificada. En 1672, por ejemplo, un vecino de Azucaica pretendía cortar algunos álamos:

"Tengo algunas alamedas dellas nezesito de sacar Doze alamos de mi propia hacienda para hazer un carro y recorrer las norias de ella" ${ }^{18}$.

Algunos años más tarde, en 1686, se solicitaba permiso para talar algunos álamos negros:

«Digo q. en la guerta de san Pablo q. es del mayorazgo que yo goço entre otros alamos negros q. ay en ella ay tres q. me causan grabe daño el uno por estar mui junto al alberca espuesto a q. un dia se le derribe y las rrayces lebanten la mas parte del alberca y los otros dos es conocido destruyen el conducto por donde biene el agua a la alberca» 19 .

De las azudas se conserva en algunos casos la alberca y el pozo (figs. 5 y 6), aunque en las afueras del pueblo, en el lugar conocido pre-

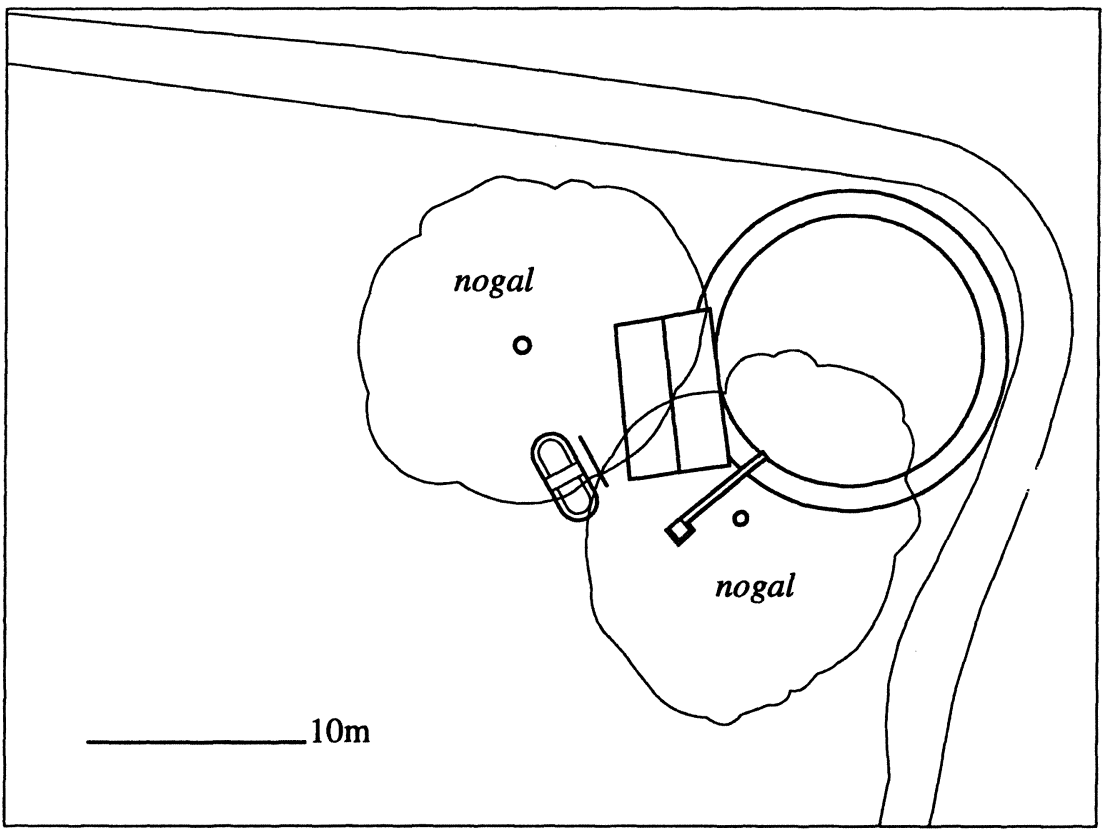

Figura 5: Alberca y pozo de noria cerca de Azucaica.

18 A. M. T., Plantíos, Caja 1.

19 A. M. T., Plantíos, Caja 2. 


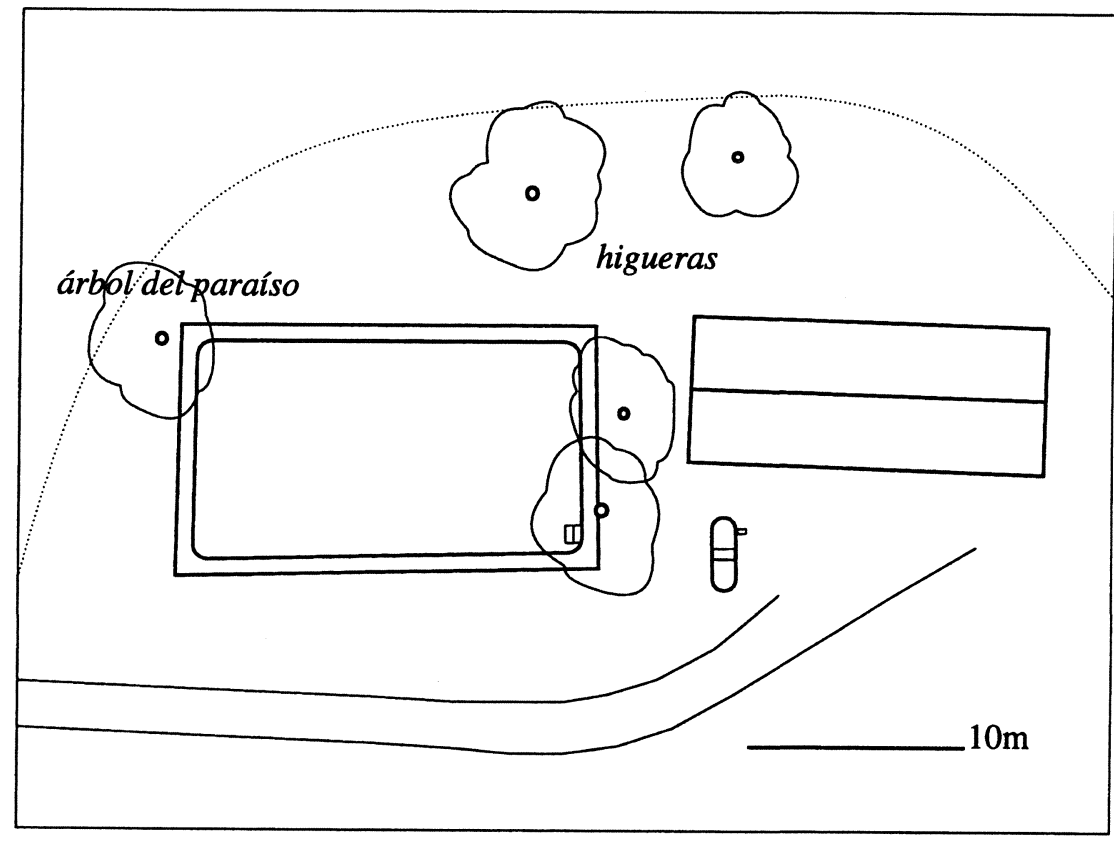

Figura 6: La Alberquilla, alberca y pozo de noria.

cisamente como La Noria, se conserva una azuda algo más completa (figs. 7 y 8). Se trata de una plataforma circular de $12,5 \mathrm{~m}$ de diámetro, elevada un metro y medio sobre el nivel del terreno y rodeada completamente de árboles ${ }^{20}$. Se intuyen restos de la rampa de subida y quedan también los machones y la viga sobre la que iba montada la maquinaria. Se conservan bien el pozo, una pequeña pila de decantación y la mitad

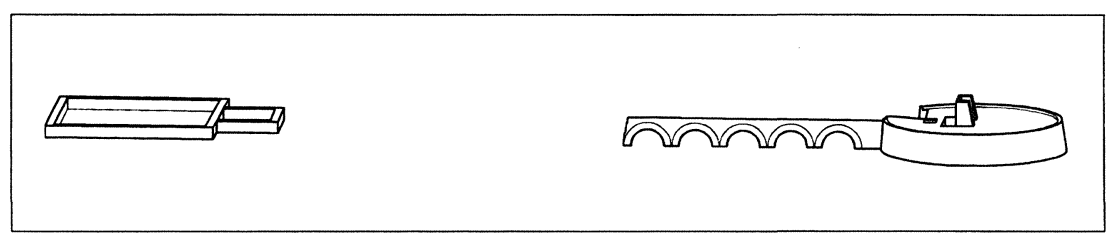

Figura 7: La Noria.

20 Ibn al-'Awwām (Sevilla, siglo xIII) recomienda en su Libro de Agricultura que para facilitar el riego se construya la noria en la cota más alta del huerto, ya sea cerca de la puerta o en medio de él. Ibn al-'Awwām, Libro de Agricultura (Kitāb al-filāha), edic. y trad. de J. A. Banqueri, Madrid, 1802, p. 144. 
de un acueducto de arcos de medio punto de 44,7 m de longitud. Este último llevaba el agua a dos albercas consecutivas, la primera menor, de 3,8 por $4,6 \mathrm{~m}$ de lado, la segunda de 11,2 por $11,1 \mathrm{~m}$. En el terreno quedan evidencias de los caballones de tierra con los que se dividiría el huerto en sectores, lo que significa que se regaba a manta ${ }^{21}$.

Es difícil decir en qué época se construyeron las norias, ya que una vez que ésta alcanzó su forma definitiva, el modelo no hacía sino repetirse. Sin embargo, algunos detalles constructivos, así como la arquitectura de ladrillo y madera de las casas de Azucaica recuerdan a la Granada morisca (fig. 9). Ya hemos visto que los moriscos trabajaron en esta zona de Toledo hasta la expulsión definitiva de 161022 .

El problema de las azudas de Azucaica es que la maquinaria de madera ha desaparecido completamente, pero según Mariano de Roa ${ }^{23}$,

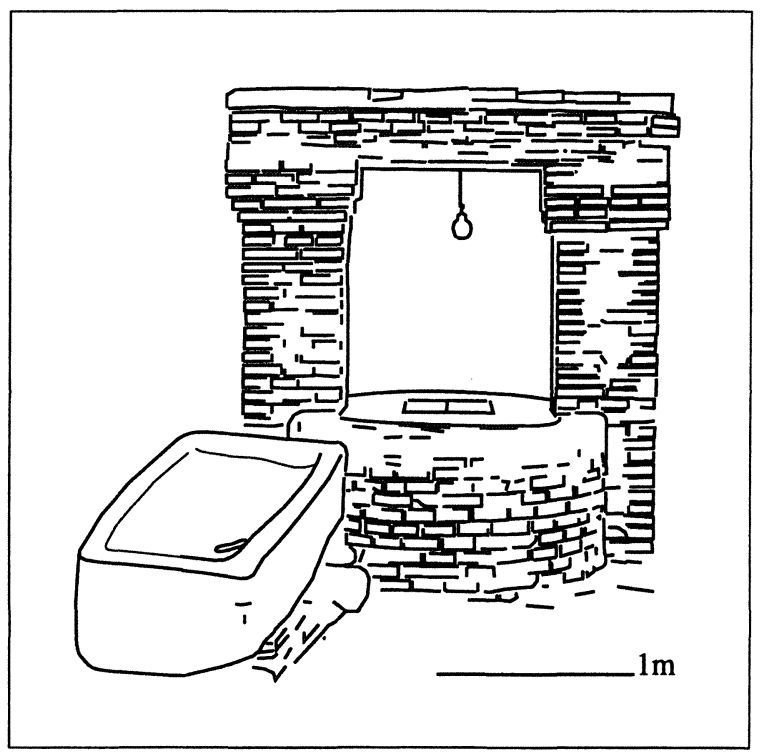

Figura 9: Pozo en un patio de Azucaica e 1/50.

21 Es notable el parecido de este ejemplo con la noria marroquí tipo que analiza G. S. Colin en el artículo citado.

22 Una anotación marginal en un documento encontrado en Mocejón señala con patética precisión: Fueronse los moriscos de Mocejón a doce días del mes de marzo de mil seiscientos diez años. Magán García, J. M., y Sánchez González, R., op. cit., p. 56.

23 Mariano de Roa vive en Azucaica y ha tenido la amabilidad de colaborar desinteresadamente en este trabajo. 
seguían el mismo modelo que la dibujada por David Roberts en Picturesque Sketches in Spain (fig. 10). Recuerda también los nombres de diferentes piezas de la noria y la madera más empleada, el álamo negro ${ }^{24}$, porque el blanco era más falso. Los arcaduces o tarrillos eran cerámi$\cos { }^{25}$, aunque con el tiempo fueron sustituyéndose por otros metálicos, y tenían un pequeño agujero en el fondo para vaciarse al parar la noria, ya que de otro modo el agua almacenada a mitad de subida se pudría. $\mathrm{El}$ animal de tiro era siempre una mula.

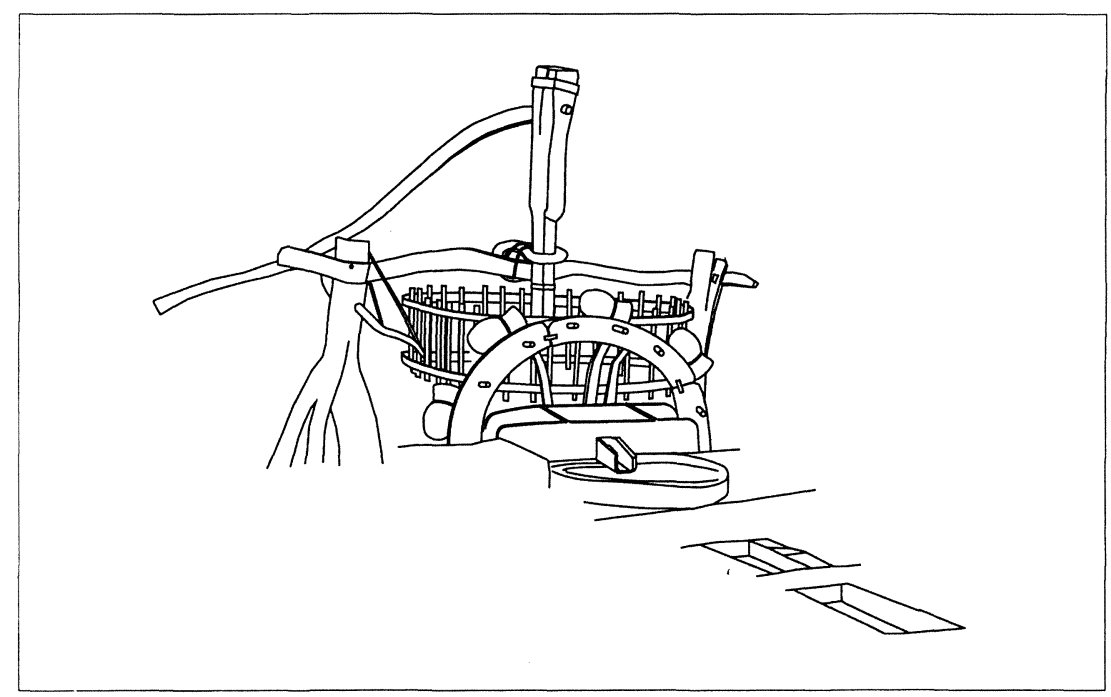

Figura 10: Noria de tiro, según D. Roberts. Calco sobre una litografía en Picturesque Sketches in Spain, Londres, 1837.

\section{LAS NORIAS DEL JARDÍN BOTÁNICO DE MADRID}

En el Jardín Botánico de Madrid se conservan también restos de una noria construida a finales del siglo XVIII ${ }^{26}$. No es extraño que tanto

${ }^{24}$ Una vez más se refiere al olmo, la madera tradicionalmente empleada en carretería.

25 Según Th. F. Glick, una noria manchega requería unos 60 arcaduces, aunque esto depende de la profundidad del pozo. Aquéllos se rompían con frecuencia debido a que la cuerda con la que se ataban a la maroma se pudría. Glick, Th. F., op. cit., pp. 647-650. Todavía hoy es fácil encontrar en Azucaica fragmentos de arcaduz alrededor de las norias.

26 Hay que destacar que los tres árboles que daban sombra a esta noria, acederaque, almez y ciclamor, se han naturalizado en nuestro país gracias a los musulmanes. 
la plataforma como el pozo tengan un diseño idéntico a los ejemplos estudiados en Azucaica, puesto que como hemos visto el modelo trascendió a sus primitivos autores o difusores, y continuó utilizándose en España hasta el mismo siglo xx (fig. 11).

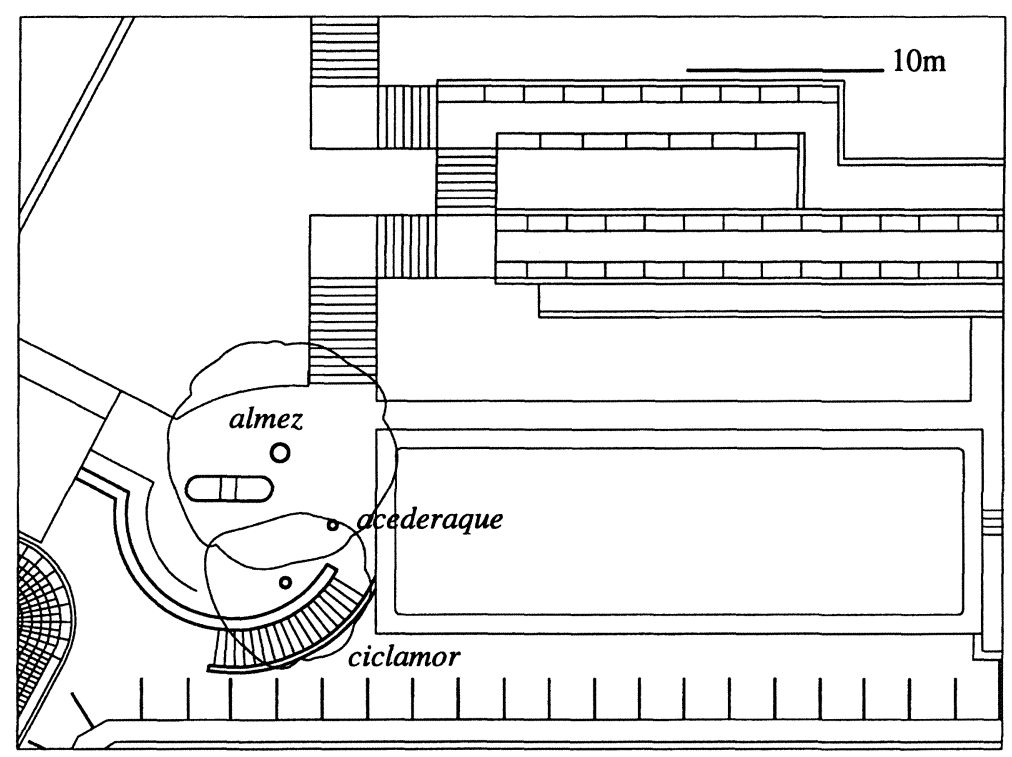

Figura 11: Noria y estanque del Real Jardín Botánico.

En el archivo del Jardín se guardan una serie de documentos que se refieren a las numerosas ocasiones en que éstas se reparaban. Esta documentación, y sobre todo las cuentas presentadas por los maestros carreteros nos van a ayudar a reconstruir la maquinaria en un caso como el de Toledo, en el que ha desaparecido todo vestigio.

En realidad, hubo en el Jardín Botánico dos norias, llamadas de arriba y abajo. En 1820 se procedía á la reparación de la rueda y cruces de la Noria de abajo del Real Jardín Botánico ${ }^{27}$. Unos meses más tarde, acausa de las muchas aguas y soles, estaban rotos el arboly el baral de la misma, por lo que hubo que avisar al maestro carretero para que los reparara ${ }^{28}$. En 1824 se llamaba de nuevo el maestro carretero por haberse desnivelado la rueda principal de la noria de avajo ${ }^{29}$, y el mismo año se

27 Archivo Real Jardín Botánico, Div. I, 29, 8, 16.

28 A. R. J. B., Div. I, 30, 1, 21.

29 A. R. J. B., Div. I, 35, 2 , 3. 
tuvieron que cambiar dos tornillos en el varal de la noria de arriva ${ }^{30}$. En 1825 hubo que hechar una abrazadera de fierro a la rueda del agua y cuatro usillos nuevos a la noria de abajo ${ }^{31}$. Y en 1828 se reparaba la lanza de la noria de arriba, los muñones de la rueda, así como las mesillas y palomillas qe. mantienen el Harteson qe. recive el agua ${ }^{32}$.

En 1833 se pensó por fin en construir un cobertizo. Ya que la maquina de la noria baja del Real Jardín Botánico ofrecía unos gastos excesivos al establecimiento por falta de un cubierto que le defendiera de la intemperie. La noria de arriba no tenía tantos problemas, precisamente porque estaba cubierta ${ }^{33}$.

En el verano era indispensable que las norias funcionaran perfectamente, sopena de perecer en pocos dias por falta de riego un considerable número de plantas que tanto había costado adquirir. La noria de arriba se agotaba al cabo de unas horas, mientras la de abajo, seguramente por estar situada en una cota más baja, tenía un mayor caudal suficiente ${ }^{34}$.

Entre 1826 y 1827 se acometió por fin una reparación general de la noria de abajo ${ }^{35}$. El maestro carretero presentó tras ello unas cuentas muy detalladas:

"Cuenta de la obra que Yo Juan de Mata Morales Maestro de Carretero tengo hecha en la Noria de avajo del Rl Jardin Botanico, por orden de la Junta directiva del mismo, con motivo de franquear su entrada al Publico el dia de San Fernando ${ }^{36}$.

Primte... Se puso la rueda del agua grande de 12 pies de diametro y a correspondencia sus gruesos

Y... Se puso la rueda Bolandera del mismo diametro

Y... Se puso 40 aguadores (fig. 12)

Y... Se puso 44 puntos

Y... Se puso las dos Cruzes de la rueda del agua

Y... Se puso dos Palomillas

Y... Se arreglaron las Cruzes al cubo viejo y se acunaron los gorrones

Y... Se puso la viga grande de 18 pies de largo.

Y... Se puso el Arbol de 16 pies de alto

Y... Se puso la rueda del aire de 12 pies de alto

Y... Y en ella se puso 44 puntos

30 A. R. J. B., Div. I, 35, 3, 2.

31 A. R. J. B., Div. I, 36, 1, 4.

32 A. R. J. B., Div. I, 41, 4, 5.

33 A. R. J. B., Div. I, 45, 5, 3.

34 A. R. J. B., Div. I, 40, 3, 3.

35 A. R. J. B., Div. I, 36, 8, 2.

36 A. R. J. B., Div. I, 40, 4, 4. 
Y... Se puso las dos Cruzes que la sostiene

Y... Se puso quatro Riostras

Y... Se puso un Jabarcon

Y... Se puso un Tejuelo de madera

Y... Se compuso todos sus herrajes y se puso una Rangua de yerro

Y... Se empego toda la Noria que se gasto tres arrobas de pez

Y... Por el trabajo de empegarla

Importa la antecedente quenta los signados tresmil seiscientos doce rl Madrid dos de Julio de 1827.»

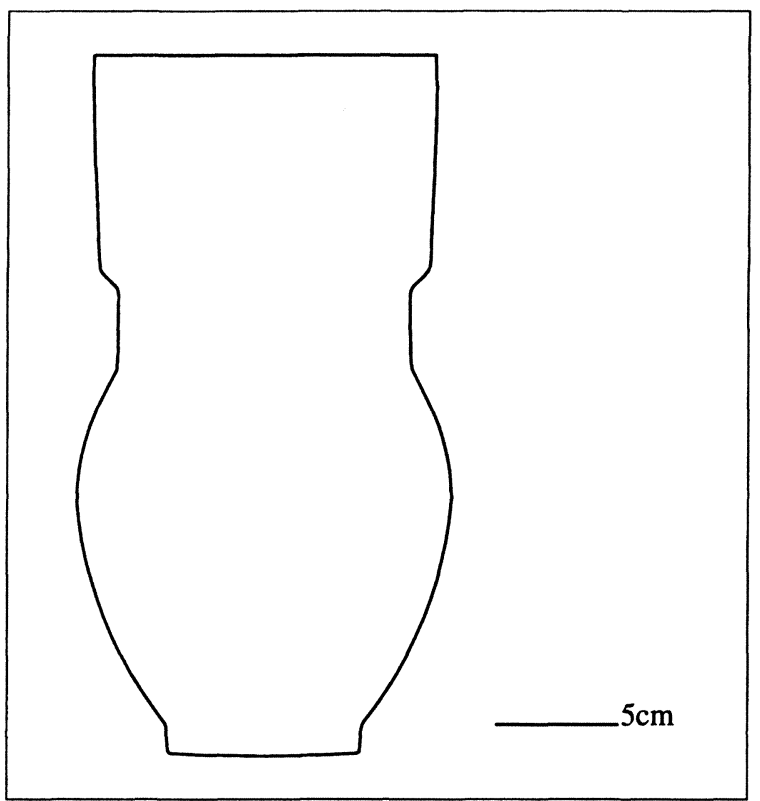

Figura 12: Arcaduz conservado en el Real Jardín Botánico e 1/4.

A partir de esta cuenta se puede deducir el proceso de montaje, e incluso una reconstrucción teórica de este modelo de noria (fig. 13). Como vemos, además de los herrajes y los arcaduces de barro cocido, la noria de abajo del Jardín Botánico constaba de más de cien piezas de madera. En primer lugar se montaría la rueda vertical, formada por la rueda bolandera y la del agua, en Azucaica de puntos o de agua, calzándola sobre el arco transversal de fábrica que aparece en todos los pozos de Madrid y Toledo. Esta rueda estaba formada por dos aros unidos por 44 puntos, y se unía al eje por dos cruces. Se montaba entonces la viga, sobre la que giraba el árbol, que terminaba en una rangua de yerro 


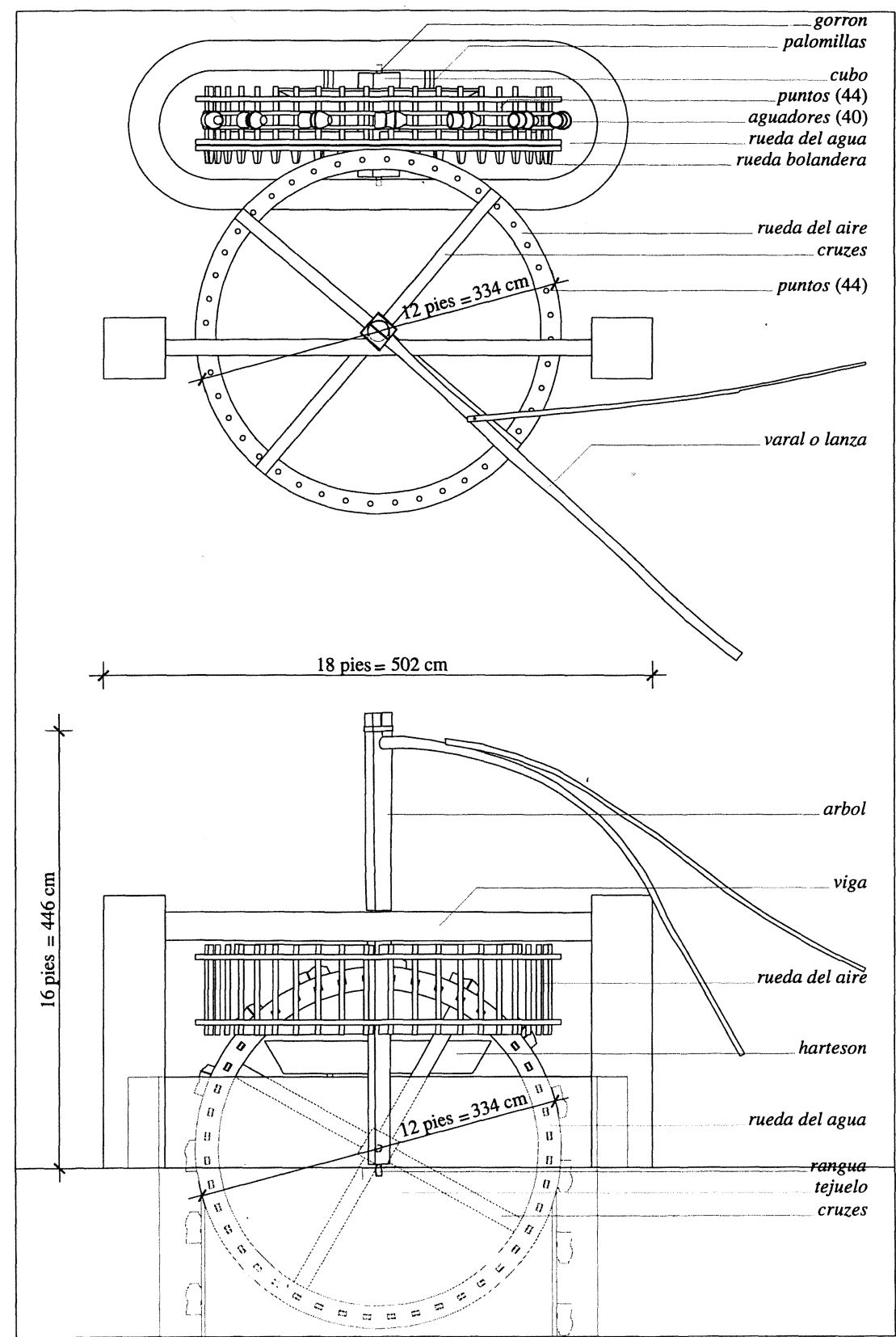

Figura 13: Reconstrucción teórica de la noria de abajo del Real Jardín Botánico, según las cuentas de 1827. 
que giraba sobre el tejuelo. El árbol era el eje vertical de la rueda del aire, en Azucaica garrucho, del mismo tamaño que la rueda del agua, y engranada a ésta por dientes que apoyaban sobre los puntos. El varal estaba conectado al árbol por encima de la viga, de modo que el animal giraba alrededor de los machones sobre los que se asentaba la máquina ${ }^{37}$. Una vez rematado todo el conjunto se empegaba toda la noria de pez para protegerla de la acción combinada del agua y el sol, y sobre la rueda de agua se montaban dos maromas paralelas de esparto, a las que se ataban los arcaduces 38 .

El animal movía este pesado conjunto de piezas gracias a que la máquina debía tener una gran inercia. Además, la desmultiplicación del esfuerzo, gracias al sistema de engranaje de las dos ruedas, tenía que ser considerable. Todo esto nos lo explica ya en el siglo XIII Ibn al-'Awwām:

«Dice Abū l-Jayr que cada dos varas de la maroma de la noria ${ }^{39}$ haya cinco arcaduces, y que quantos mas fueren los dientes en la rueda pequeña que juntamente con la mayor hace circular la dicha maroma, vendrá á ser esta máquina mas liviana y ligera, y lo mismo si el palo atravesaño fuese largo; el qual no perjudica sea de treinta ó casi treinta palmos. Que tambien se da fácil curso á la maroma doble con cortar del palo derecho lo que sobresale por encima del horado del atravesaño; y lo mismo si las piezas donde estriban los arcaduces fueren de madera pesada; á cuyo fin han de hacerse mas gruesas y pesadas de lo acostumbrado, respecto á que así se logra aquel efecto. Dicen, que para estorbar el encuentro de las gradas de la escalerilla con los arcaduces en el agua del pozo se haga á cada uno de estos en el fondo un pequeño agujero, con lo qual no ladeándose, estan libres de quebrarse unos con otros, ó en la expansión del pozo; los quales vaciándose tambien al parar la maroma, por esta causa dura la misma mucho tiempo" ${ }^{40}$.

37 La otra solución posible es que lo haga por debajo de la viga, pero en este caso, a pesar de que la solución constructiva del nudo es mejor, la viga debe tener unas dimensiones enormes.

38 Un curioso tratado de mecánica del siglo xvi, Los Veinte y Un Libros de los Yngenios y Maquinas se refiere también a este empegado: bien se sabe que las maderas son offendidas y gastadas de las aguas, y que el remedio para ella es la pez y el çufre mezclado y untadas q' se conservan. Pseudo-Juanelo Turriano, The Twenty-one Books of Devices and of Machines, introd. de A. García-Diego, Madrid, 1984, vol. II, p. 449.

39 En este caso utiliza la forma sãniya.

40 Ibn al-'Awwām, op. cit., pp. 146-147. 


\section{CONCLUSIÓN}

Con la ayuda del ejemplo de Madrid podemos imaginar las azudas de Azucaica, ya que como sabemos, las dos tendrían un diseño ya establecido, utilizando una fórmula en cierto modo anacrónica, standard (fig. 14).

La noria podía llegar a suministrar un caudal de $1 \mathrm{l} / \mathrm{s}$ si contaba con un solo animal de tiro, y aproximadamente $1,75 \mathrm{l} / \mathrm{s}$ si eran dos. Es evidente que una máquina de estas características no puede competir con una bomba moderna, que a la misma profundidad da con facilidad 5 o $61 / \mathrm{s}$.

Sin embargo, como elemento fundamental de toda una cultura tradicional de regadío que durante siglos transformó el paisaje de nuestro país, aunque sea sólo desde un punto de vista teórico, hemos querido contribuir aquí a su recuperación.

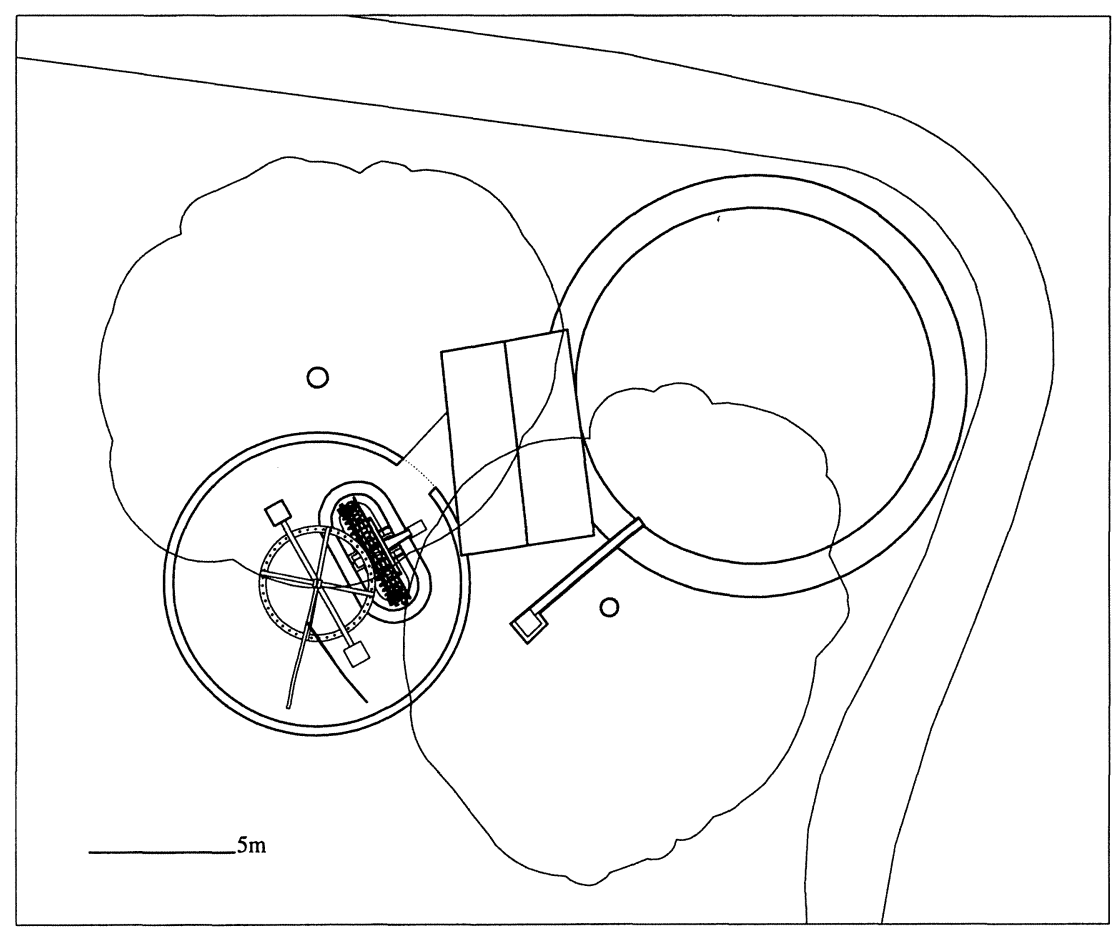

Figura 14: Reconstrucción de una de las norias de Azucaica. 


\title{
RESUMEN
}

La noria de tiro es un artefacto utilizado para elevar agua de un pozo con la ayuda de una caballería. Este tipo de noria es frecuente en todas las regiones secas de la Península Ibérica. Este trabajo estudia su lugar de origen y fecha de introducción en la Península, así como la influencia de términos árabes en el vocabulario castellano. Se analizan también los restos de noria encontrados por el autor en Azucaica (Toledo), así como la conservada en el Real Jardín Botánico de Madrid. A pesar de que la fecha de construcción en un caso y otro diverge en cientos de años, se demuestra que la semejanza entre los dos grupos es notable. Este hecho, sumado a la existencia de una serie de documentos conservada en el Real Jardín Botánico, permite una reconstrucción teórica en el caso de Toledo.

\begin{abstract}
The noria de tiro (drawn water-wheel) is a device which is used to raise water from wells with the aid of a draught animal. This type of noria is common to all of the arid regions of the Iberian Peninsula. This study is an investigation into the origins of this type of noria, the date of its introduction into the Peninsula and the influence of Arabic terminology for the noria on Castilian vocabulary. This article also discusses two examples: the remains of a noria discovered by the author in Azucaica (Toledo), and the noria preserved in the Real Jardín Botánico of Madrid. Although the dates of construction of the two examples diverge by hundreds of years, the similarities between them are striking. In addition, a group of documents preserved in the archive of the Real Jardín Botánico helps to theoretically reconstruct the Toledan noria.
\end{abstract}

